\title{
Hereditary spastic ataxia simulating disseminated sclerosis
}

\author{
MOHSEN MAHLOUDJI \\ From the Nemazee Hospital, Shiraz Medical Centre, Shiraz, Iran
}

In 1929 Ferguson and Critchley published a detailed clinical study of a family, many members of which suffered from a disease resembling disseminated sclerosis. As far as Ferguson and Critchley could discover the syndrome described had not been recorded previously.

The average age of onset was 35 to 45 years and the initial symptoms were weakness and unsteadiness of the legs, followed by dysarthria, visual disturbances, dysaesthesiae of the arms and legs, and bladder disorders. The syndrome progressed steadily without remissions. The duration of the disease in fatal cases was from five to 10 years and, in those still living at the time, up to 16 years. There were alterations in the emotional sphere with marked euphoria and instability of mood. Optic atrophy was present in some and in a few Argyll Robertson pupils were noted; one patient had a positive Wassermann reaction. A marked feature in a majority of patients was a defect of upward movement of the eyes. Pyramidal signs were present in most of them and the cerebellar type of incoordination in several. Speech defect was noted in almost every case, mainly due to cerebellar dysfunction. Extrapyramidal signs formed a prominent feature in many of the patients. Sensory disturbances were present in the majority, diminution to light touch and pin prick being more marked than postural and vibratory sensibility. Gait was markedly abnormal in every patient and in the final stages they were unable even to stand. Frequency and precipitancy of micturition, leading to occasional incontinence, were observed in some patients.

An Iranian family is now described with a large number of members suffering from a syndrome with marked similarities to the one described by Ferguson and Critchley. Four affected members were seen personally and information obtained about others.

CASE 1 A 38-year-old widow from Teheran was admitted to the Nemazee Hospital in April 1961 with the chief complaints of difficulty in walking for six years and dysarthria for four years. She had been quite well up to the age of 32 when she first noticed difficulty in running and jumping which gradually deteriorated until, at the time of admission, she could hardly walk unaided. Later there was involvement of the upper limbs making ordinary movements hard to carry out. The speech was affected later still and, although comprehensible after four years, was markedly abnormal. There was no history of any visual disturbances in the past and she had never had any pain or paraesthesiae. The sphincters had always been under full control.

Examination revealed an intelligent, cooperative and pleasant woman of average stature. She had an ataxic, scanning speech with a nasal twang. The optic discs, although on the pale side, were considered to be within normal limits. Visual acuity was $20 / 40$ in each eye and could be corrected to normal with minus one $(-1.00)$ lenses. The fields showed slight peripheral contraction but no scotoma was detected. Upward and lateral conjugate movements of the eyes were defective but there was no nystagmus. There was an economy of facial expression and the muscles were thought to show some weakness. The jaw jerk was brisk. No other cranial nerve deficit could be detected.

In the upper limbs, the tone of muscles was normal and the power quite good. There was moderate cerebellar ataxia of both sides. The arm jerks were brisk and equal. No sensory loss of any modality was noted.

In the lower limbs there was considerable global increase in tone but only slight weakness of dorsiflexion and toe movements bilaterally. Severe and widespread incoordination was a marked feature. The tendon jerks were very brisk and equal but there was no clonus. Both plantar responses were extensor. The abdominal reflexes were present and equal. Vibration sense was lost at and below the left knee and much reduced on the right side. Otherwise there was no sensory deficit and position sense was intact. She was unsteady when standing with the feet together and this did not change on shutting the eyes. The gait was extremely slow, on a wide base and markedly ataxic. She was unable to jump off the ground.

Investigations including radiographs of the chest, a full blood count, urine and stool analysis, the blood sugar and urea, and an electrocardiogram, were all within normal limits. However, serological examination for syphilis on the first occasion showed a positive venereal diseases research laboratory test at a titre of 1 in 32 and a Kahn titre of 1 in 4 . A second examination four days later proved them both to be negative. Apparently a similar result had been obtained elsewhere and she had been given penicillin which had to be stopped after two 


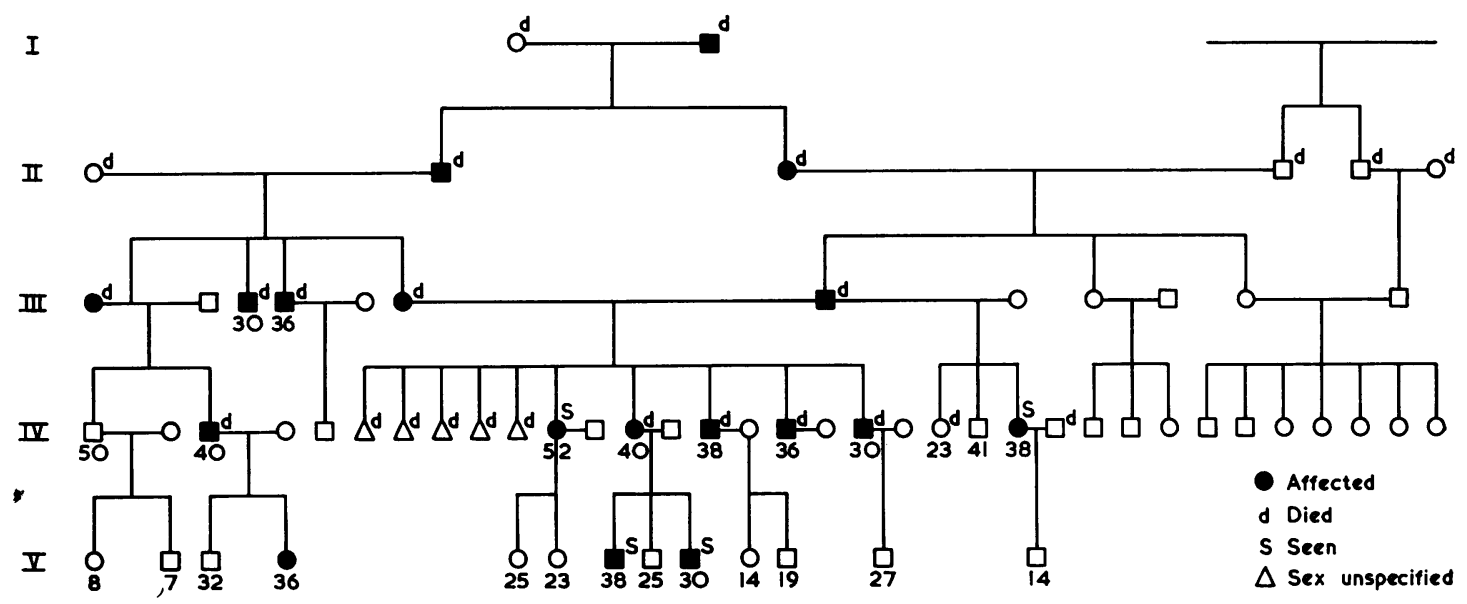

FIG. 1.

days because of skin sensitivity. The possibility of syphilis in the husband had been raised but there was no proof of it; he had died of alleged cerebral vascular disease before the age of 40 years.

Because a similarly afflicted relative had had a lumbar puncture in Paris 20 years previously and this was said to have made him much worse, she refused to have the cerebrospinal fluid examined. Apparently a very distinguished Parisian neurologist had warned against lumbar puncture in any of the affected members.

Figure 1 shows the pedigree. The case just described is IV, 23. Another affected member, IV, 11, the only living step sister of case 1 , was seen in her own home.

CASE 2 A 52-year-old woman from Teheran had been well up to the age of about 32 when she developed difficulty in walking and a year or two later had become dysarthric. At about the same time movement of the hands had become awkward. She had gradually deteriorated until at the time of examination she was unable to walk at all.

Examination revealed a thin woman who looked older than her age. Although fully orientated and fairly cooperative, she showed some resentment at being examined and was rather suspicious. The speech was markedly ataxic and only on listening carefully was it comprehensible.

The optic discs were pathologically pale but there was no demonstrable defect of visual acuity. The external ocular movements were full and there was no nystagmus.

In the upper limbs, the tone and power of muscles appeared normal but there was gross cerebellar ataxia. All the tendon reflexes were very brisk. There was no sensory deficit except for slight diminution of vibration sense at and below the elbows.

In the lower limbs, there was some increase of tone in muscles below the knees but the power appeared normal. There was severe ataxia and she was unable to walk at all and found standing difficult. The reflexes were all extremely brisk and unsustained ankle clonus was obtained on both sides. The plantar responses were sharply extensor. On full sensory testing only reduction in vibration sense of equal distribution was noticed throughout the limbs.

CASE 3 A 38-year-old man $(\mathrm{V}, 7)$ was seen at a later date with the chief complaints of difficulty in walking and unsteadiness of gait for about two years. There had been steady deterioration until at the time of examination he was unable to run.

Examination revealed a man of average intelligence who tended to minimize his disability. In fact he had not sought medical advice voluntarily but had been persuaded by a kind friend of the family to come to the clinic. He insisted that he did not suffer from the family disease but a minor separate illness.

His speech appeared to be normal. Visual acuity, as judged by reading newspaper print, was quite good and fundoscopy revealed no abnormality. There was a defect of upward conjugate movement of the eyes but otherwise the cranial nerves were intact.

In the upper limbs the tone and power of muscles were normal and there was only slight incoordination. The reflexes were brisk and equal and there was no sensory deficit.

In the lower limbs there was marked global spasticity with unsustained ankle clonus but no loss of power. Coordination was moderately defective and all the tendon jerks were markedly exaggerated and equal. The left plantar response was extensor and the right flexor. The abdominal reflexes were absent. Once again the only sensory deficit was diminution of vibration sense below the knees. His gait was spastic and he had difficulty in jumping off the ground.

Of the other living affected members only V, 9 was seen. He was a man of about 30 who had started with similar symptoms at about the age of 28 , but he minimized his disabilities and did not care to see 
a doctor. From his walking and the few words of greeting he uttered on arrival, there was no doubt about the staggering and spastic gait and the dysarthric speech.

A so far unaffected member, V, 5, the elder daughter of case 2 , was a very intelligent and reliable girl who helped in compiling the information about the whole family. She showed understandable concern and was naturally worried about her younger sister who, against all advice, still planned to marry a first cousin, V, 8. She had observed her mother and other affected relatives closely and the summary of her findings, as a lay person, is as follows:

Almost all of them started to show symptoms at or about the age of $\mathbf{3 0}$ years. In her mother and aunt (cases 1 and 2) symptoms, on the whole, appeared late. In some of them the first symptom was a little difficulty in swallowing fluids when they would cough but without regurgitation through the nose. This was not seen in the three patients examined. The involvement of the lower limbs was an early sign, first noticed on jumping over gutters and walking in a straight line. A year or two later dysarthria would become evident and the upper limbs would show ataxia. The picture would then progress to the full syndrome as in the cases described.

According to the informant, there were undoubted mental changes. They tended to minimize their disabilities and their morale usually remained high. They showed nervousness and paranoid tendencies but never became confused or disorientated. Death usually occurred about the age of 40 and case 2 held the record in longevity. The actual cause of death could not be surmised.

\section{DISCUSSION}

It is clear that in isolation any of the patients personally examined might well have been suffering from disseminated sclerosis. It is now generally accepted that there is a familial incidence of this disease in at least $10 \%$ of all examples but it has not yet been suggested, and still less proved, that there is any genetic basis for this disorder.

Perusal of the pedigree, and assuming it to be valid, seems to make it quite certain that this is a progressive, fatal and degenerative disease of the central nervous system. Again if the information be valid this syndrome seems to be determined by a single autosomal dominant gene, probably of great rarity. The disorder described seems to approximate only to that previously observed by Ferguson and Critchley (1929), and the differences are small.
Such differences are frequent in hereditary disorders of the nervous system when, not infrequently, the disorder may run true to type in any one family though no two families present an identical picture. Both Ferguson and Critchley (1961) are of the opinion that nothing similar to the syndrome they first described has been recorded since.

The gene concerned is obviously not sex-linked and is dominant. Patient III, 6, had married twice, the first wife being a first cousin (III, 5). That marriage produced 10 children of whom five died in infancy or early childhood (ages 1 to 4 years). There were no known miscarriages and all except one (case 2) died before the age of 41 years. It is probable that the ones who died in childhood had received a double dose of the gene but it should not be forgotten that the infant mortality rate is high in Iran and was undoubtedly higher in those days. Therefore, a dominant gene represented by A (for example Aa $\mathrm{x}$ Aa in the cousin marriage) easily fits the whole picture except for the second filial generation (marriage of II, 1 and II, 2). All their children appear to have been affected and, on the basis of probability, the chances of that happening is no higher than $4 \%$. In the absence of more data no explanation is offered.

Regarding the last generation, attention must be drawn to the fact that the syndrome starts at about 30 years of age and some of them may well develop it in the years to come.

Unfortunately first cousin marriages are extremely common in Iran and, as I hope to show in future articles, a phenomenal number of cases of hereditary neurological disease of all varieties are encountered in ordinary practice. The impending marriage of cousins V, 8 and V, 6 will no doubt produce results worthy of a paper in the next century.

\section{SUMMARY}

A very rare hereditary syndrome of spastic ataxia, closely resembling disseminated sclerosis, is described in an Iranian family. The pedigree shows that it is determined by a single autosomal dominant gene.

I am much indebted to Dr. Hugh Garland, who first drew my attention to the nature of the condition described, for his constant encouragement and helpful advice. I am also grateful to Zhaleh for all her help.

\section{REFERENCES}

Critchley, M. (1961). Personal communication. Ferguson, F. R., and Critchley, M. (1929). Brain, 52, 203. - (1961). Personal communication. 